\title{
RisK OF CERVICAL TRANSForaminal EPIDURAL INJECTIONS By ANTERoIr Approach
}

To the Editor:

I would like to share my recent experience with an anterior approach to a cervical transforaminal epidural. This was attempted in a 56-year old white male with left-sided neck pain and upper extremity pain in a C6 dermatomal pattern. His MRI showed a left C5,6 herniated nucleus pulposus extending into the neuroforamen with associated foraminal narrowing and nerve root compression. This patient underwent a previous left C5,6 transforaminal epidural steroid injection with anterior approach with excellent relief without any difficulty or complications. Due to the recurrence of the symptoms, a second injection was planned.

The patient was brought to the operating room for a repeat injection. This was performed identically to the first injection using an anterior transforaminal approach. A 25 -gauge $3 \frac{1}{2}$ " quincke point spinal needle was utilized for the procedure. Using tunnel vision, the needle was directed toward the "J-point". After contacting the articular process, the needle was carefully walked anteriorly into the posterior aspect of the neuroforamen at its mid-aspect. The C-arm (OEC-9600) was then placed in an AP view and the needle advanced to the mid-facet line. Needle position was confirmed in 2 views and then contrast was injected under live fluoroscopic imaging. Contrast (Omnip- aque $240 \AA$ ) was seen to spread immediately into a vascular structure with rapid wash out. The spread of this contrast was into a fine vascular network within the spinal canal. The needle was repositioned and the same spread was seen. The needle was backed out and repositioned this time with spread outside the foramen; the needle was advanced and contrast reinjected but again the same vascular structure filled with rapid washout (Figs. 1, A to $C$ ). At that juncture the transforaminal approach was stopped and the patient was turned prone for a posterior midline approach at C6,7. This was accomplished without incident (Fig. 2). There was no adverse sequela from this procedure.

It was the impression of the physician that this vascular spread most likely represented an intraarterial injection given the distribution of the contrast spread and the speed with which the contrast washed out. All contrast injections in this case were performed under live fluoroscopic imaging. Given the rapid washout seen after contrast injection it is very likely that this would have been missed if the injection had not been performed under live imaging. (Note: after washout there was some residual contrast within the foramen, which may have led an unsuspecting operator to inject their steroid mixture at that point).

There have been multiple cases in the United States in the last several years of severe complications following cervical transforaminal epidural steroid injections with almost all the patients demonstrating permanent, severe, neurologic damage (paralysis, coma, and/or death). The most commonly proposed theory is that particulate steroid is injected into a feeding artery of the spinal cord, which lies within the neuroforamen. The particulate steroid then blocks the microcirculation leading to spinal cord infarction. Others have suggested an alternative mechanism such as vasospasm related to the injection. Recommendations to date have included using blunt tip needles to avoid vascular puncture (however this requires a larger needle, an even larger introducer, and it is a difficult needle to steer) and the use of non-particulate steroids (a recommendation that we have adopted) (1-4).

This case demonstrates one potential mechanism as to how these dreaded complications might occur following a cervical transforaminal epidural steroid injection and emphasizes the need for the interventional pain physician to utilize extremely careful technique when performing this procedure. It is this author's opinion that the injection of contrast under live fluoroscopic imaging is essential to maximally ensure that there is no intravascular uptake given the small caliber of these vessels and the speed with which
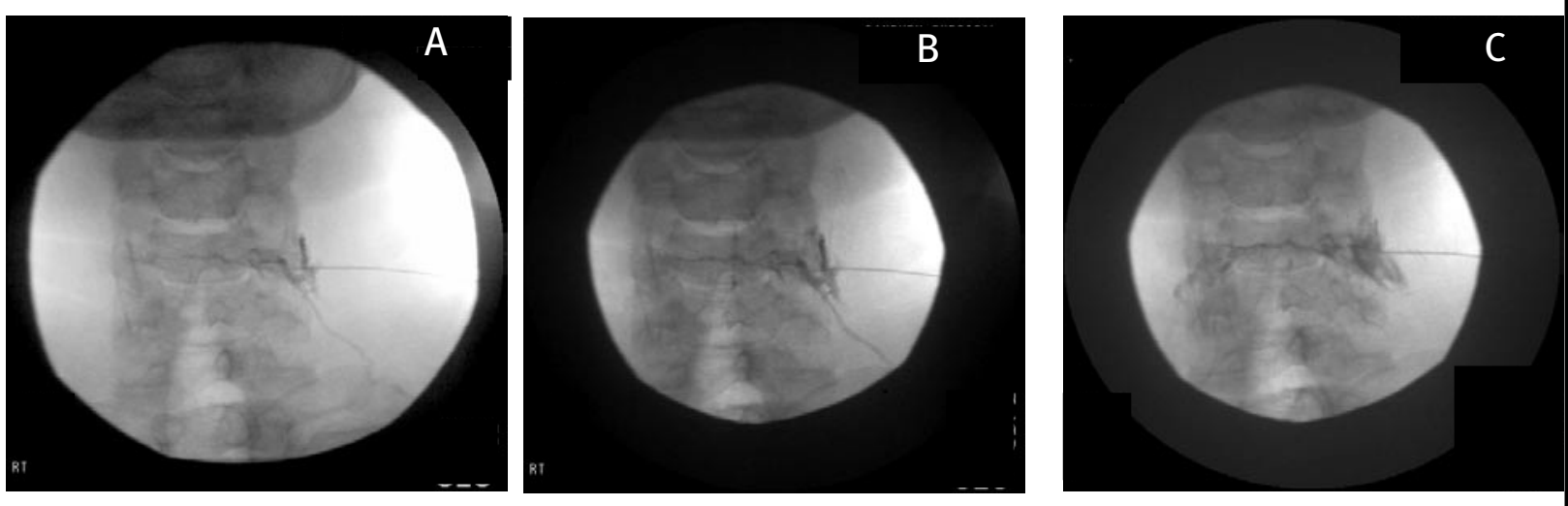

Fig. 1: A to $C$ show the anterior approach for a transforaminal epidural injection, along with injection of contrast rapid washout and filling of the fine vascular network. 


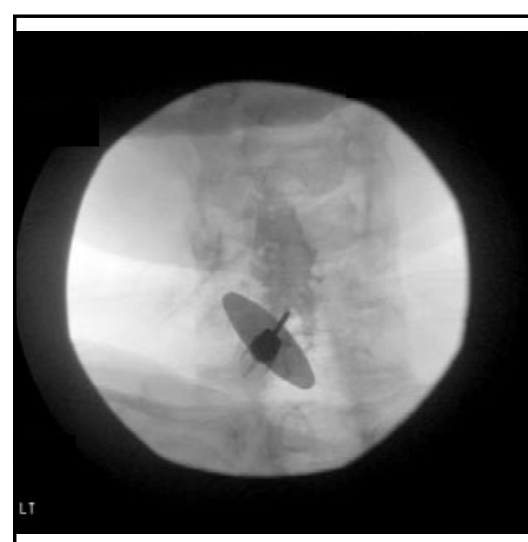

Fig. 2. Posterior approach to interlaminar cervical epidural injection washout is seen. While there is no guarantee that this can prevent such devastating complications, this technique may improve the safety and lower the risk. The author uses low volume, 12" tubing ( 0.3 $\mathrm{mL}$ vol.).

\section{REFERENCES}

1. Baker R, Dreyfus P, Mercer S et al. Cervical transforaminal injection of corticosteroids into a radicular artery: A possible mechanism for spinal cord injury. Pain 2003; 103:211-215.

2. Houten JK, Errico TJ. Paraplegia after lumbosacral nerve root block: Report of three cases. Spine / 2002; 2:70-75.
3. Nelson JW. Letter to the Editor. In response to Houten JK, Errico TJ. Paraplegia after lumbosacral nerve block. Spine J 2003; 2:88-89.

4. Racz GB, Manchikanti L, Schultz D, Kloth D, Dunbar E, Trescot A, Helm S, Hansen HC, Jasper JF. Discussion on Safety of Interventional Techniques. ASIPP's Cadaver Workshop, Memphis, TN, October 25-27, 2002, May 17-18, 2003.

David S. Kloth, MD

Medical Director

Connecticut Pain Care, PC

109 Newton Road

Danbury CT 06810

E-mail: dkmd@ctpaincare.com 